\title{
Planar Hall Effect in Magnetite (100) Films
}

\author{
Xuesong Jin, Rafael Ramos*, Y. Zhou, C. McEvoy and I.V. Shvets \\ SFI Nanoscience Laboratories, School of Physics, Trinity College Dublin, Dublin 2, Ireland
}


Abstract.

Giant Planar Hall effect (GPHE) has been observed in epitaxial magnetite (100) films grown on $\mathrm{MgO}$ substrates. The effect is manifested as jumps in the transverse resistivity when the film is subjected to a swept, in-plane magnetic field. The jumps are two orders of magnitude higher than previously observed in metallic ferromagnets. Recently, the same effect has been reported for other materials, but unlike our results, they present GPHE at low temperature only. The magnitude of the GPHE observed at room temperature has potential applications such as magnetic sensors and nonvolatile memory elements.

Keywords: Planar Hall Effect, Anisotropic Magnetoresistance, Magnetite. 


\section{INTRODUCTION}

The planar Hall effect (PHE), like the anisotropic magnetoresistance (AMR), has its origin in the magnetization-related anisotropy of resistivity of the ferromagnetic materials, both being different manifestations of the same underlying phenomenon. In the ferromagnetic films, the resistivity depends on the angle between the magnetization $\mathbf{M}$ and the current density $\mathbf{J}$. For a single domain film, the electric field is given by ${ }^{1}$ :

$$
\begin{gathered}
E_{x}=j \rho_{\perp}+j\left(\rho_{\|}+\rho_{\perp}\right) \cos ^{2} \theta \\
E_{y}=j\left(\rho_{\|}-\rho_{\perp}\right) \sin \theta \cos \theta
\end{gathered}
$$

where $\mathrm{j}$ is the current density applied in the $\mathrm{x}$ direction, $\theta$ is the angle between $\mathbf{M}$ and the $\mathbf{J}, \rho_{\perp}$ and $\rho_{\|}$are the resistivity values perpendicular and parallel to $\mathbf{M}$, respectively. The AMR phenomenon is described by the first equation (1), while equation (2) describes the PHE.

The PHE was previously employed in ferromagnetic metals, as a tool to study inplane magnetization ${ }^{2,3}$, out-of-plane magnetization ${ }^{4}$ and it has also been studied in lowfield magnetic sensor applications ${ }^{5}$. Recently, a large switching signal in the PHE has been measured in $(\mathrm{Ga}, \mathrm{Mn})$ As thin film devices and $\mathrm{LaSrMnO}_{3}$ films ${ }^{6,7}$, this effect is observed to be 4 orders of magnitude higher than previously observed in ferromagnetic metals and it is called the giant planar hall effect (GPHE). Unlike the previous reports, where the GPHE was observed at low temperature, in this study we have measured GPHE jumps at room temperature, in our case the effect is two orders of magnitude larger than in the ferromagnetic metals. The magnitude of the switching signal measured at room temperature could have potential applications in spintronics, such as field sensors and non-volatile memory elements. 


\section{EXPERIMENT}

The $100 \mathrm{~nm} \mathrm{Fe}_{3} \mathrm{O}_{4}$ film, with double cross structure, was grown on $\mathrm{MgO}$ (100) substrate with a size of $10 \times 10 \mathrm{~mm}^{2}$ using oxygen plasma assisted molecular beam epitaxy (MBE), system (DCA MBE M600), with a base pressure of less than $5 \times 10^{-10}$ Torr. The substrates were annealed at $600^{\circ} \mathrm{C}$ for $1 / 2 \mathrm{~h}$ in UHV and $2 \mathrm{~h}$ in an oxygen atmosphere. The deposition was done by means of e-gun evaporation from Iron pellets ( $99.995 \%$ pure) in a plasma oxygen environment of $1.1 \times 10^{-5}$ Torr and a substrate temperature of $250{ }^{\circ} \mathrm{C}$ at a growth rate of $0.2 \AA / \mathrm{s}$. The double cross structure was fabricated using a shadow mask.

The longitudinal and transverse resistances were investigated using the four probe method, with a Keithley 2400 sourcemeter and a switching system to perform almost simultaneous measurement. A bias current of $100 \mu \mathrm{A}$ was applied along the (100) direction. An external in-plane magnetic field was applied using an electromagnet with a range up to $1 \mathrm{~T}$ and a resolution of $0.1 \mathrm{Oe}$. The field orientation was changed to measure the angular dependence. Temperature dependence was also investigated using a closed cycle refrigerator $(\mathrm{CCR})$.

The longitudinal resistance, $\mathrm{R}_{\mathrm{xx}}$, was measured between $\mathrm{B}$ and $\mathrm{C}$ (inset Fig. 1). The transverse resistance, $\mathrm{R}_{\mathrm{xy}}$, was obtained by measuring the resistance between $\mathrm{A}$ and C. Due to the low value of $R_{x y}$ in comparison to $R_{x x}$, there is a significant contribution of the longitudinal component over the transverse one, which was corrected using the formula: $R_{x y}^{\text {corr }}(H)=R_{x y}(H)-\left(R_{x y}(0) / R_{x x}(0)\right) \cdot R_{x x}(H)$. 


\section{RESULTS AND DISCUSSION}

The resistivity of thin films as a function of temperature was measured to evaluate the stoichiometry of the films. A clear jump in resistivity (so-called Verwey transition) is observed around $115 \mathrm{~K}$ which is close to the one in single crystal $(120 \mathrm{~K})^{8}$. From the presence of the Verwey transition we could conclude that the film's stoichiometry is close to that of ideal magnetite. The detailed characterization of the magnetite film could be found in Ref 9. The AMR in the $\mathrm{Fe}_{3} \mathrm{O}_{4}$ films was investigated first. A constant inplane magnetic field (2T) was applied, the longitudinal resistance $\left(\mathrm{R}_{\mathrm{xx}}\right)$ and the transverse resistance $\left(\mathrm{R}_{\mathrm{xy}}\right)$ were measured as a function of the angle between the magnetic field and the current (see Fig. 1). At such a high field the magnetization is expected to be parallel to the applied field. We found that $\mathrm{R}_{\mathrm{xx}}$ shows $a \cos ^{2} \theta$ dependence and $\mathrm{R}_{\mathrm{xy}}$ follows a $\sin \theta \cos \theta$ dependence which is consistent with the AMR theory for a single domain ferromagnetic film ${ }^{1}$.We can see that the presence of Antiphase Boundaries (APBs) still does not alter the single-domain state-like behaviour of the film. APBs are an intrinsic consequence of the nucleation and growth mechanism in the film ${ }^{10}$. They arise from the fact that the $\mathrm{Fe}_{3} \mathrm{O}_{4}$ lattice parameter is nearly twice that of $\mathrm{MgO}$, as a result there are half lattice shifts between nucleation islands. Furthermore, the rotational symmetry of the film is lower than that of the substrate. APBs alter the exchange interaction between grains within the film.

We have measured GPHE for magnetite at room temperature. Figure 2 shows the $\rho_{\mathrm{xx}}$ and $\rho_{\mathrm{xy}}$ for a magnetic field sweep and at an orientation of $\theta=45^{\circ}$. The jumps observed in the longitudinal resistivity $\left(\rho_{\mathrm{xx}}\right)$ and in the transverse resistivity $\left(\rho_{\mathrm{xy}}\right)$, are close to the coercive field values for the magnetite film, thus it is thought that this effect 
arises from the magnetization rotation, which could be determined by the applied magnetic field, the anisotropy field of the film and the antiferromagnetic interaction across the APBs. The PHE jumps $\left(\Delta \rho_{x y}^{\text {jump }} \equiv \rho_{x y}^{o .1 T}-\rho_{x y}^{\text {peak }}\right)$ are in the order of $\mu \Omega \mathrm{cm}$, two orders of magnitude higher than previously observed in metallic ferromagnets ${ }^{11,12}$.

The temperature dependence was also studied, which is of importance in elucidating the underlying physical mechanisms. $\Delta \rho_{x y}^{j u m p}$ and $\Delta \rho=\rho_{\|}-\rho_{\perp}$ are represented as a function of temperature (see Fig. 3a), $\Delta \rho$ measurements are in good agreement with those previously observed in $\mathrm{Fe}_{3} \mathrm{O}_{4}{ }^{13}$. Opposite to $\Delta \rho, \Delta \rho_{x y}^{\text {jump }}$ increases with decreasing temperature, thus implies that the GPHE temperature dependency cannot be explained by the conventional AMR theory. The ratio $\Delta \rho_{x y}^{j u m p} / \rho_{x x}(0 T)$ should provide information about the carrier polarization. In $(\mathrm{Ga}, \mathrm{Mn}) \mathrm{As}$ thin film devices, this ratio tracks the magnetization of the sample ${ }^{6}$. Our measurements show that this ratio decreases with temperature (see Fig. 3b), this behavior is contrary to what was observed for the magnetization of magnetite film in the same range of temperatures ${ }^{14}$. It is thought that the effect of the antiferromagnetic interaction across the APBs may play an important role in the PHE of films. The antiferromagnetic coupling and the exchange frustration across APBs is expected to become stronger with decreasing temperature. This factor may contribute to the temperature dependence of the $\Delta \rho_{x y}^{\text {jump }}$ in the films.

\section{CONCLUSION}

We observed the GPHE in magnetite at room temperature. The size of the effect, two orders of magnitude larger than previously observed for ferromagnetic metals, has 
potential applications in spintronics. The angular and thermal dependence of the PHE jumps was studied. The GPHE is thought to be a response to the magnetization rotation, which would be influenced by the applied field, the anisotropy field and the exchange interaction through the APBs. Further studies need to be performed to clarify the detailed effect of the APBs.

\section{Acknowledgements}

This work was supported by Science Foundation Ireland (SFI) under Contract No. 00/PI.1/C042. 


\section{References:}

${ }^{1}$ D. A. Thompson, L. T. Romankiw and A. F. Mayadas, IEEE Trans. Magn. 11, 1039 (1975).

${ }^{2}$ G. Li, T. Yang, Q. Hu, H. Jiang, and W. Lai, Phys. Rev. B 65, 134421 (2002).

${ }^{3}$ S. Das, H. Yoshikawa, and S. Nakagawa, J. Appl. Phys. 93, 8098 (2003).

${ }^{4}$ F. Y. Ogrin, S. L. Lee and Y. F. Ogrin. J. Magn. Magn. Mater. 331-339, 219 (2000).

${ }^{5}$ A. Schul, F. Nguyen Van Dau and J. R. Childress, Appl. Phys. Lett. 66, 2751 (1995).

${ }^{6}$ H. X. Tang, R. K. Kawakami, D. D. Awschalom and M.L. Roukes, Phys. Rev. Lett. 90, $107201(2003)$.

${ }^{7}$ Y. Bason, L. Klein, J. B. Yau, X. Hong and C. H. Ahn, Appl. Phys. Lett. 84, 2593 (2004).

${ }^{8}$ Ciaran McEvoy, Xuesong Jin and I. V. Shvets, J. Magn. Magn. Mater. 283, 171 (2004).

${ }^{9}$ Xuesong Jin, Ciaran McEvoy and I. V. Shvets, J. Magn. Magn. Mater. 286, 128 (2005).

${ }^{10}$ D. T. Margulies, F. T. Parker, M. L. Rudee, F. E. Spada, J. N. Chapman, P. R. Aitchison and A. E. Berkowitz. Phys. Rev. Lett. 79, 5162 (1997).

${ }^{11}$ B. Zhao, X. Yan and A. B. Pakhomov. J. Appl. Phys. 81, 5527 (1997).

${ }^{12}$ S. U. Jen, K. P. Huang and J. C. Lee. J. Appl. Phys. 84, 843 (1998).

${ }^{13}$ M. Ziese and H. J. Blythe, J. Phys.: Condens. Matter. 12, 13 (2000).

${ }^{14}$ M. Ziese, R. Höhne, H.C. Semmelhack, K.H. Han, P. Esquinazi and K. Zimmer. J. Magn. Magn. Mater. 331-342, 279 (2004). 


\section{Figure captions}

Fig. 1. Longitudinal (AMR) and transverse (PHE) resistance as a function of the angle. The magnetization is considered to be parallel to an external applied field (2T).

Fig. 2. Longitudinal $\left(\rho_{\mathrm{xx}}\right)$ and transverse $\left(\rho_{\mathrm{xy}}\right)$ resistivity for a swept, in-plane magnetic field. The field orientation is at $\theta=45^{\circ}$. Arrows indicate the scan direction.

Fig. 3. (a) PHE jump and AMR dependence with temperature. (b) GPHE to resistivity ratio as a function of temperature. 


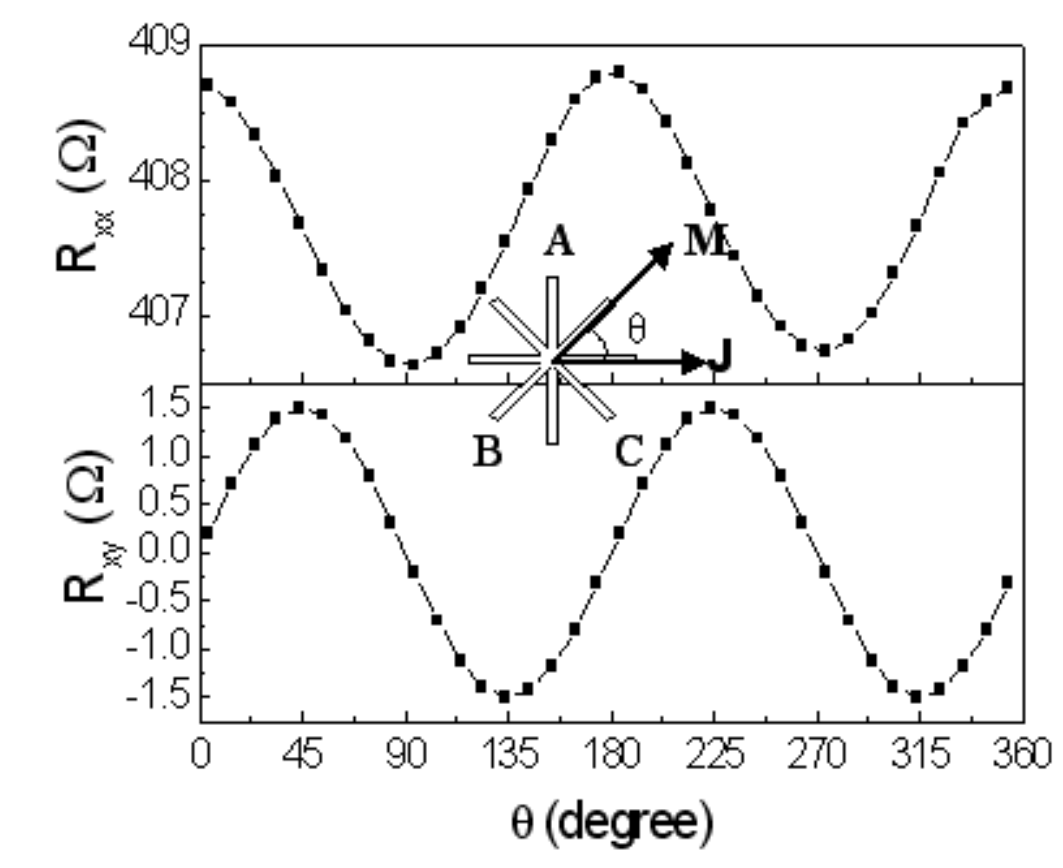




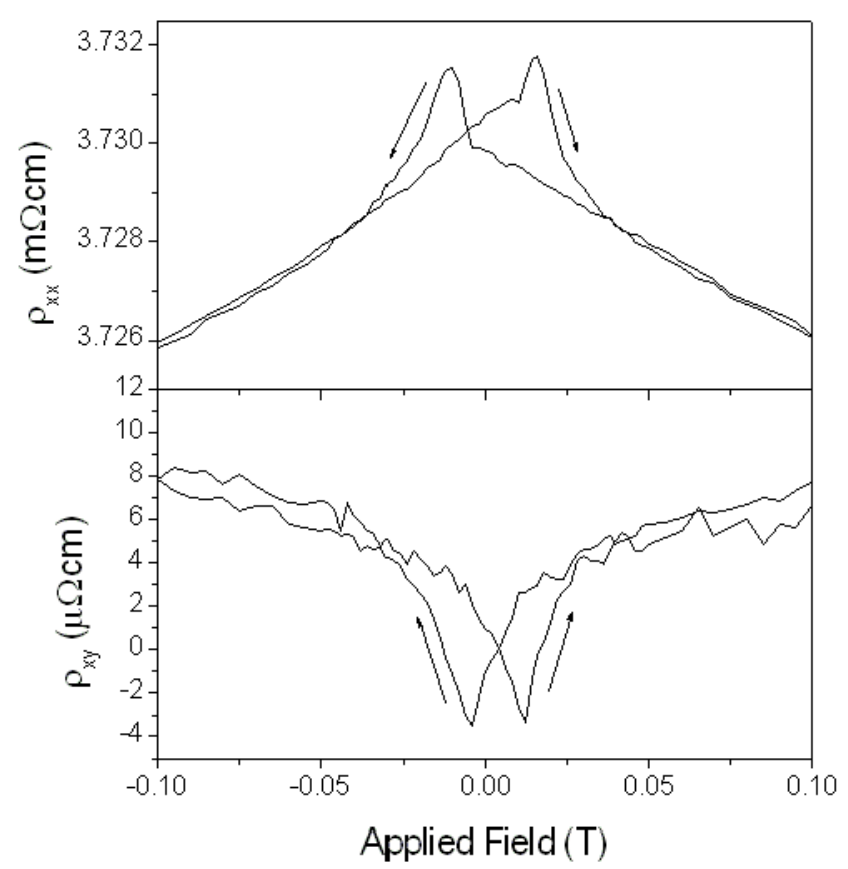




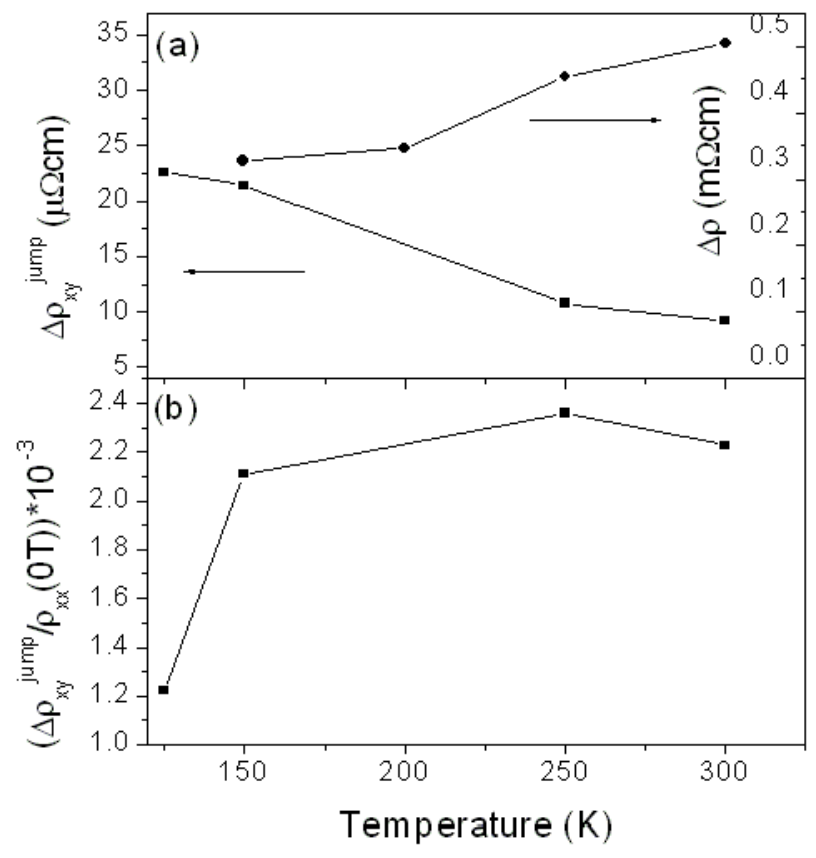

